\title{
Laboratory Scale Investigation of Dispersion Effects on Saltwater Movement due to Cutoff Wall Installation
}

\author{
Masahiro Takahashi ${ }^{1}$, Kazuro Momii ${ }^{2}$ and Roger Luyun $\mathrm{Jr}^{3}$ \\ ${ }^{1}$ Nippon Koei Co. Ltd., Tokyo, Japan \\ ${ }^{2}$ Kagoshima University, Kagoshima, Japan \\ ${ }^{3}$ University of the Philippines Los Baños, Laguna, Philippines
}

\begin{abstract}
In the numerical investigation of saltwater transport in coastal aquifers, we need to correctly evaluate the hydrodynamic dispersion in the flow field. In this study, we focused on the role of dispersivity in the removal process of residual saltwater in a laboratory scale cutoff wall experiment. From a pulse-type fluorescent tracer injection experiment in a saturated porous media of glass beads with a mean diameter of $0.088 \mathrm{~cm}$, the estimated longitudinal and transverse dispersivities were found to be $0.07 \mathrm{~cm}$ and $0.0025 \mathrm{~cm}$, respectively. Numerical analysis of the saltwater intrusion and subsequent removal after cutoff wall installation using SEAWAT and the generated dispersivity ratio $\left(\alpha_{\mathrm{L}} / \alpha_{\mathrm{T}}\right)$ of 28 reproduces well the measured salt concentration changes with time. Whereas, if a dispersivity ratio of 10 is used in the numerical simulation, transverse dispersion in the saltwater and freshwater mixing zone becomes large and the residual saltwater is removed faster than the laboratory experiment. Inversely, if 100 was used, the residual saltwater removal time took longer. The transverse dispersion is a key parameter in the mechanical dispersion of saltwater in the mixing zone after cutoff wall installation.
\end{abstract}

\section{INTRODUCTION}

Among several countermeasures to prevent saltwater intrusion in coastal aquifers, artificial subsurface barriers store and control groundwater and ensure a consistent extraction of freshwater without causing seawater intrusion. Luyun et al. (2009) showed experimentally and numerically that residual saltwater trapped upon installation of these cutoff walls gradually retreated before being completely removed from the reservoir behind the cutoff wall. However, they observed slight differences in the time for complete removal between the experimental and numerical results. To numerically investigate the seawater movement, we need to correctly evaluate the dispersion parameters in the flow field. In this study, we focused on the role of dispersivity in the saltwater transport in the laboratory scale cutoff wall experiment. The objectives of this study are to determine the longitudinal and transverse dispersivities of a porous medium using fluorescein tracer test and to demonstrate their influence on saltwater movement after cutoff wall installation.

\section{MATERIALS AND METHODS}

\section{Experimental setup}

We conducted our experiments in a transparent flow tank with internal dimensions of $60 \mathrm{~cm}$ length, $30 \mathrm{~cm}$ height, and $1 \mathrm{~cm}$ thickness, sufficiently thin to approximate quasi 2dimensional system (Figure 1). A 5-mm diameter injector tube used for pulse tracer injection is located $10 \mathrm{~cm}$ from the left saltwater reservoir and $5 \mathrm{~cm}$ from the bottom of the flow tank. Beside it, $5 \mathrm{~cm}$ from the left reservoir and $5 \mathrm{~cm}$ from the bottom, a 2-mm diameter electric 
conductivity (EC) sensor is used to measure the salt concentration change with time during cutoff wall experiments. We packed the main flow tank with homogeneous glass beads with a nominal diameter of $0.88 \mathrm{~mm}$ to model an unconfined aquifer. The freshwater and saltwater reservoirs were separated by fine mesh screens to each side of the glass bead section. The heads in the reservoirs were controlled by adjustable drainage pipes.

\section{Tracer calibration and pulse experiments}

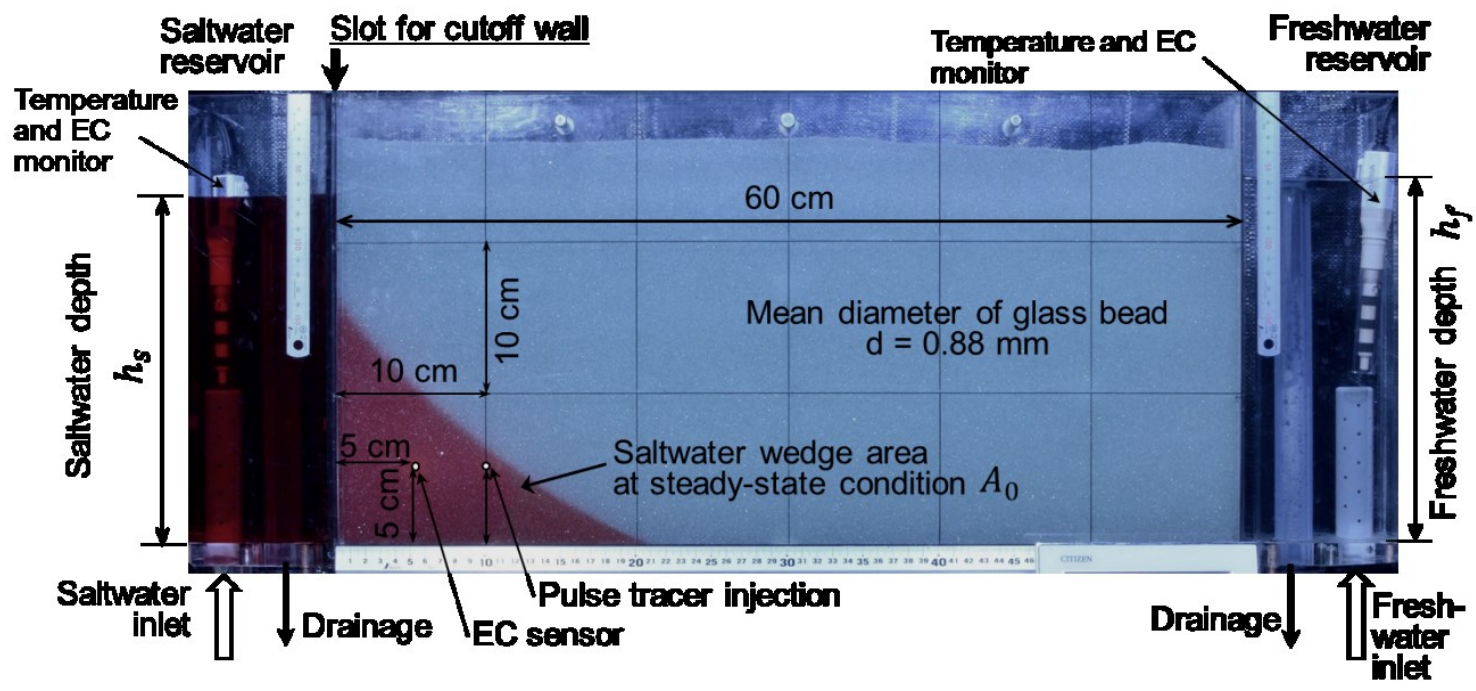

Figure 1. Experimental set-up.

We conducted tracer experiments to determine the longitudinal dispersivity, $\alpha_{\mathrm{L}}$, and transverse dispersivity, $\alpha_{\mathrm{T}}$, of the porous medium. We chose the fluorescein sodium salt $\left(\mathrm{C}_{20} \mathrm{H}_{10} \mathrm{Na}_{2} \mathrm{O}_{5}\right)$ as a tracer. When a fluorescent dye tracer is exposed to ultra-violet light, the dye emitted visible light in proportion to the tracer concentration (Huang et al. 2002). We used two 40W Black Light Blue ultraviolet (UV) tubes as a light source at a distance of 80 $\mathrm{cm}$ inside a dark room to excite the fluorescent tracer. Images were recorded using a Nikon D5100 digital camera.

A tracer calibration test of the setup was first conducted to determine the relationship between the tracer concentration and luminosity. We saturated the porous medium with known concentrations of fluorescein salt $\left(C=0,0.2,2,5,10,15 \mathrm{mg} \mathrm{L}^{-1}\right)$ and took photographs under UV light in the darkroom. We used the image processing software, Image J (U. S. National Institutes of Health, USA) to split the RGB (Red, Green, and Blue color bands) components and determine the luminosity values of the different concentrations using pixel value statistics. One pixel is equivalent to $0.024 \mathrm{~cm}$. The Green color band shows very good correlation with tracer concentration, so we used this relationship to convert luminosity to actual concentration in the pulse injection tests.

We then performed a pulse tracer test with the water level at the left and right reservoirs set to $23.5 \mathrm{~cm}$ and $23.0 \mathrm{~cm}$, respectively. The tracer solution with $15 \mathrm{mg} \mathrm{L}^{-1}$ concentration was introduced over the full thickness of the porous medium by using the injector. We injected a slug of $1.5 \mathrm{mg}$ fluorescein salt for a period of $60 \mathrm{~s}$ using an automatic syringe pump to ensure a constant injection rate. As the tracer plume moves in the direction of flow, images were then captured by the digital camera. All these were done inside the dark room. We processed the captured images using Image $J$ and with the derived relationship between $G$ 
luminosity values and concentration, we determined the concentration profiles of the tracer plume at times equal to $0 \mathrm{~min}, 10 \mathrm{~min}$, and $29 \mathrm{~min}$ after the 1 -min tracer injection.

\section{Cutoff Wall Experiments}

We prepared saltwater by dissolving commercial salt in water and dyed it with a red edible pigment (Food dye red No. 102 New Coccine). Small peristaltic pumps were used to constantly supply saltwater and freshwater from supply buckets below the setup to the left and right reservoirs, respectively. Diluted saltwater overflowing from the water level adjustment cylinder on the drains into an independent channel while freshwater falls back into the freshwater bucket and recirculated into the system by the pumps. The cutoff wall experiment procedure was similar to Luyun et al. (2009). Initially, the porous medium and reservoirs were filled with freshwater. The water levels were set to a constant head of $h_{f}$ $=24.0 \mathrm{~cm}$ on the freshwater side and $h_{s}=23.0 \mathrm{~cm}$ on the saltwater side (Figure 1). When the flow stabilized, a shutoff wall was inserted to isolate the saltwater reservoir and the freshwater in it was then replaced with the red saltwater solution. The saltwater intrusion process was initiated with the removal of the shutoff wall. Density driven flow progressed until steady state was achieved. After steady state was established, a 20-cm cutoff wall was inserted into the slot and the movement of the residual saltwater wedge was recorded by a digital camera. We measured the areas of the attenuating saltwater wedge at different time intervals. The experiment ended when the saltwater wedge was completely flushed out.

\section{Numerical Simulations}

In the pulse tracer experiments, we used MODFLOW and MT3DMS (Zheng and Wang 2008) to simulate the concentration profiles developed from the image analysis. A uniform grid spacing of $0.48 \mathrm{~cm}$ was used. Using all parameters from the experiments, we assumed several paired values of longitudinal and transverse dispersivities. The paired values that give the minimum root mean squared difference (RMSD) between concentration profiles determined from image analysis and those from the simulation, were considered to be the valid values of the longitudinal and transverse disperivities in our experiment.

We used the SEAWAT Ver4 (Langevin et al. 2008) to simulate the 20-cm cutoff wall experimental condition and using the estimated longitudinal and transverse dispersivities. Additionally, we performed the simulations using dispersivity ratio $\alpha_{\mathrm{L}} / \alpha_{\mathrm{T}}$ of 10 and 100 . We then compared the changes in concentration of the saltwater wedge as measured at the EC probe location during the experiment and the computed values at the same location from the simulation results. The purpose of the simulation is not only to reproduce the experimental results but also to demonstrate the effects of dispersivity on the saltwater removal process due to the cutoff wall installation.

\section{RESULTS AND DISCUSSION}

\section{Pulse Tracer Experiments}

Figure 2 shows a comparison of the tracer concentration profiles along the horizontal axis from the injection point. The longitudinal dispersivity, $\alpha_{\mathrm{L}}$, and transverse dispersivity, $\alpha_{\mathrm{T}}$, values that gave the minimum RMSD to the plotted concentration profiles from image analysis, were determined to be $0.07 \mathrm{~cm}$ and $0.0025 \mathrm{~cm}$, respectively. The concentration profiles (black lines) calculated using MODFLOW and MT3DMS from these derived values are shown to be in good agreement with the experimental values. The dipersivity ratio then is $\alpha_{\mathrm{L}} / \alpha_{\mathrm{T}}=28$, which is within the range of 10 and 100 given by Todd and Mays (2005). 


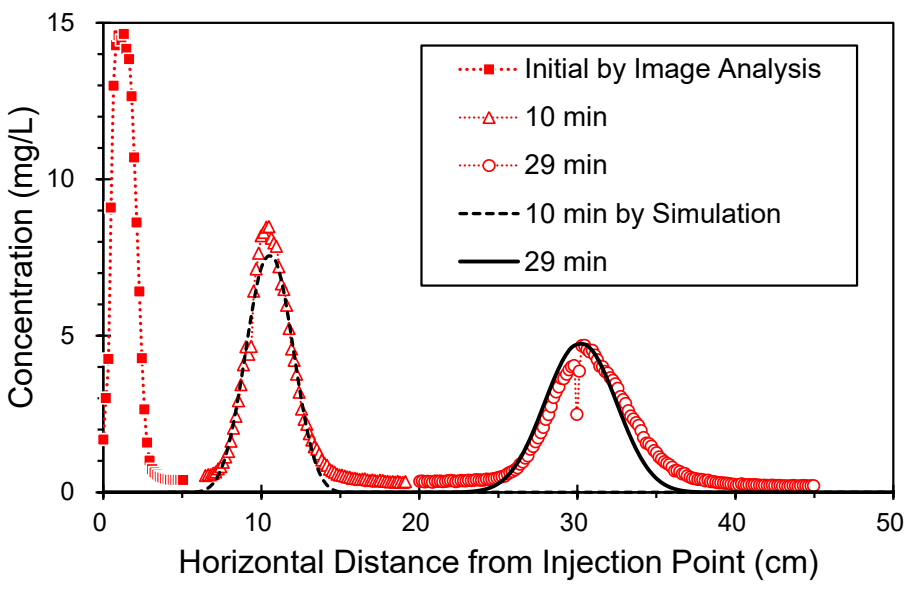

Figure 2. Comparison of the tracer concentration profiles along the horizontal axis at $t=0$ min (initial), $10 \mathrm{~min}$ and 29 min after the pulse tracer injection.

\section{Cutoff Wall Experiments}

Figure 3 presents the comparison of the experimental and the numerical results at (a) initial steady state, and (b) $12 \mathrm{~h}$ after installation of the $20-\mathrm{cm}$ cutoff wall. The numerical model predicted well the overall outline of the saltwater wedge. Dispersive flux of salt is carried with the freshwater as it flows along the mixing zone and over the cutoff wall. Saltwater is transported by diffusion and dispersion and then carried to the outlet by the freshwater flow. Since the cutoff wall prevented additional saltwater supply, there is a net flux of salt flowing out over the wall. The saltwater wedge area slowly but continuously decreased from $A_{0}=138 \mathrm{~cm}^{2}$ at initial steady state to $A_{12}=38 \mathrm{~cm}^{2}$ and $A_{24}=7 \mathrm{~cm}^{2}$ after 12 and $24 \mathrm{~h}$, respectively. In the experiment, all the saltwater was flushed out after $t_{\max }=33 \mathrm{~h}$ (Figure 4).

The freshwater velocity profiles (Figure 3) near the freshwater reservoir at the initial steady state and after the cutoff wall installation did not change. This supported the experimental measurement that the freshwater discharge towards the saltwater reservoir remains constant during the saltwater removal process after the cutoff wall installation. In general, the freshwater velocity near the discharge zone is smaller than that above the saltwater wedge toe location because the cross-sectional area of freshwater flow decreases gradually towards the seaside discharge zone from the toe location.

Comparing the attenuating saltwater wedge versus time, Figure 4 shows that the experimental and numerical results are in good agreement. The reduction rate of the saltwater wedge area gradually becomes slower with time. As the saltwater wedge area reduces due to the saltwater removal, the cross-sectional area of freshwater flow above it increases and the freshwater velocity decreases. Smaller velocity results in smaller dispersion and the dilution of the saltwater across the mixing zone between the saltwater and freshwater is weakened. Thus, it takes more time to remove the residual saltwater with time after the cutoff wall installation. Dispersion is a key parameter to the dilution of the saltwater in the mixing zone during saltwater removal. 
(a) Steady state

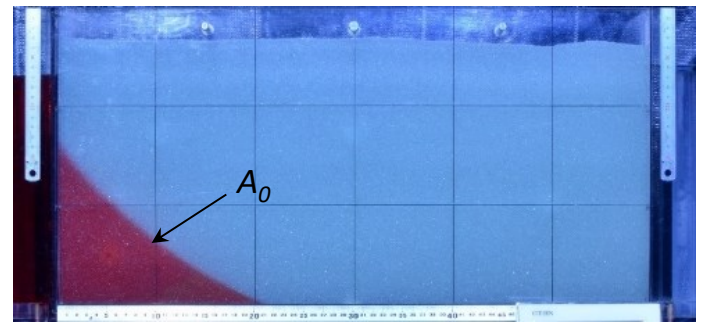

(b) 12 hours after the cut-off wall installation

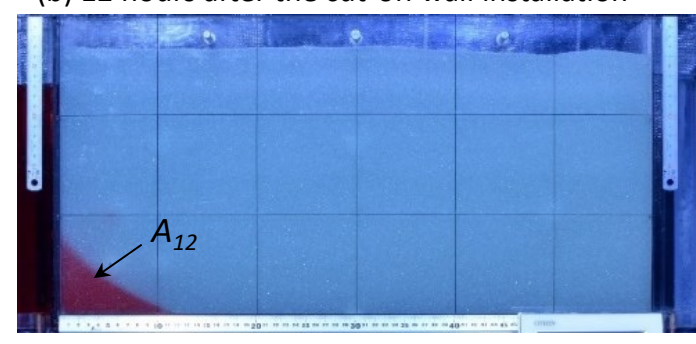

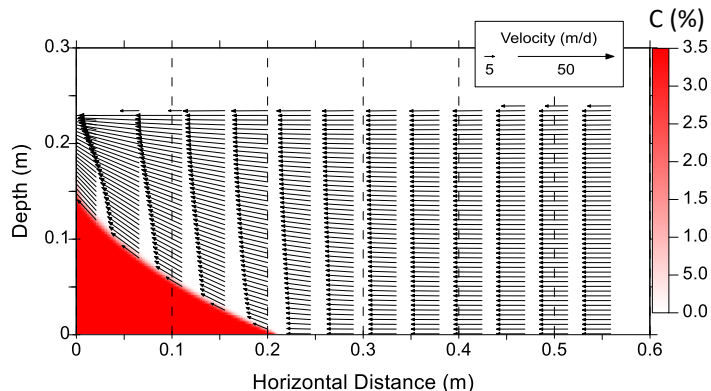

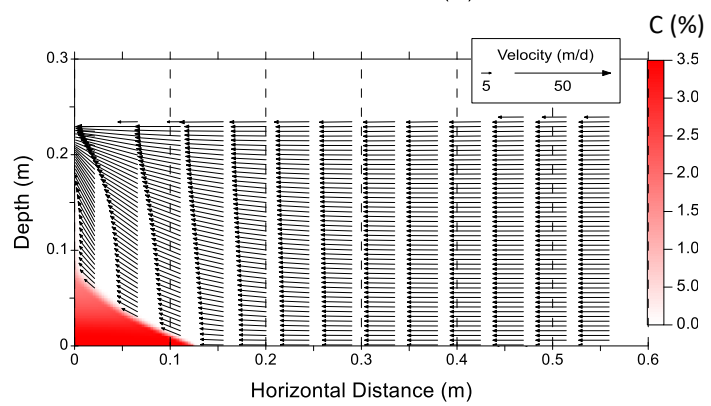

Figure 3. Comparison between the experiments and numerical simulations.

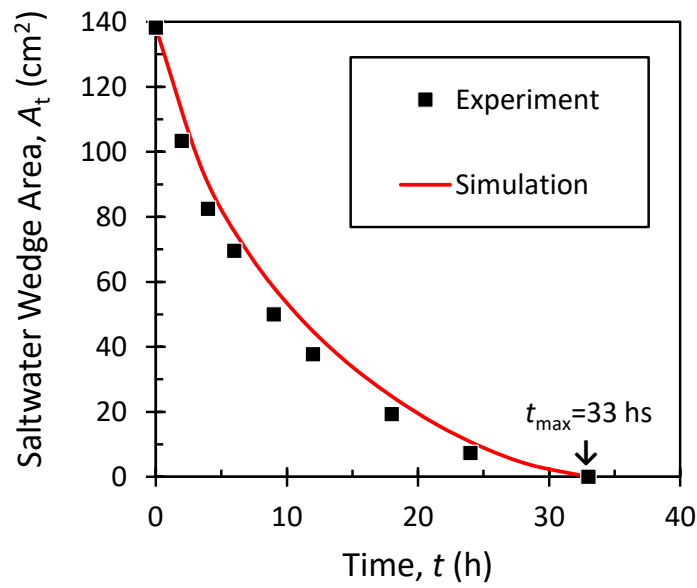

Figure 4. Experimental and numerical results of reduction in saltwater wedge area.

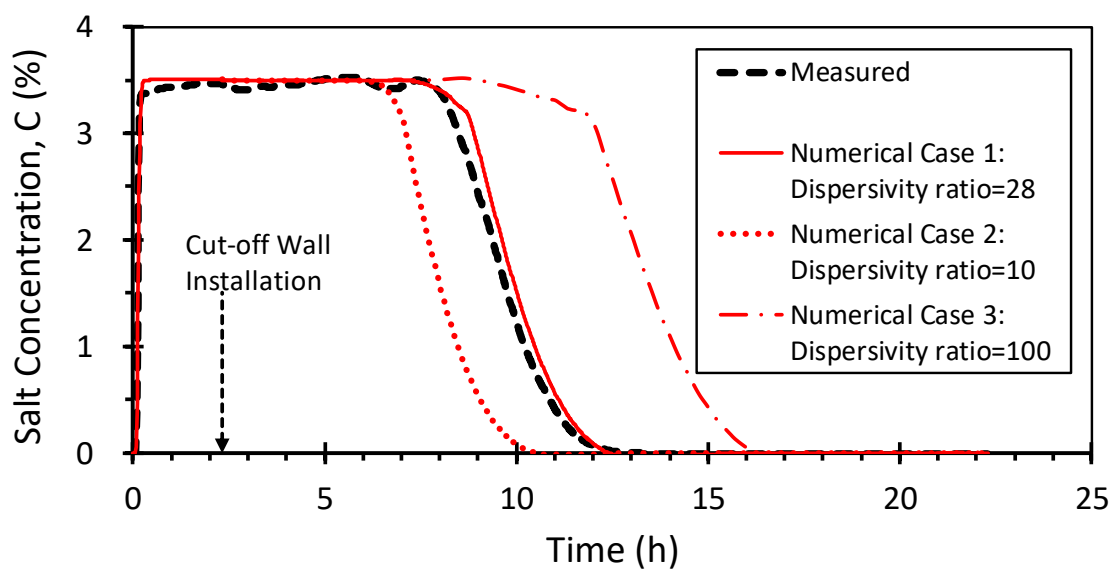

Figure 5. Measured and numerical results of salt concentration changes during saltwater intrusion process and subsequent removal after cutoff wall installation. 
Figure 5 compares the measured concentration of the attenuating saltwater wedge with three cases of model computations using different dispersivity ratios: (a) the experimental Case 1, $\alpha_{\mathrm{L}} / \alpha_{\mathrm{T}}=28\left(\alpha_{\mathrm{L}}=0.07 \mathrm{~cm}, \alpha_{\mathrm{T}}=0.0025 \mathrm{~cm}\right) ;(\mathrm{b})$ the hypothetical Case $2, \alpha_{\mathrm{L}} / \alpha_{\mathrm{T}}=10\left(\alpha_{\mathrm{L}}=0.07\right.$ $\left.\mathrm{cm}, \alpha_{\mathrm{T}}=0.007 \mathrm{~cm}\right)$, and; (c) the hypothetical Case 3: $\alpha_{\mathrm{L}} / \alpha_{\mathrm{T}}=100 \quad\left(\alpha_{\mathrm{L}}=0.07 \mathrm{~cm}, \alpha_{\mathrm{T}}=0.0007\right.$ $\mathrm{cm})$. Note that the value of longitudinal dispersivity, $\alpha_{\mathrm{L}}$, is fixed. During the intrusion process, there is no apparent difference in the salt concentration. Upon installation of the cutoff wall, however, it can be observed that only the concentration change in Case 1 agreed well with that of the experiment. For Case 2, the saltwater wedge attenuated and was completely flushed out faster than in the experiment. For a constant longitudinal dispersivity, the lower the ratio the higher is the transverse dispersivity and the broader is the mixing zone of the saltwater wedge. This means that it will be easier for the dispersed flux of salt to be carried away by the freshwater discharge as it flows along the mixing zone and over the cutoff wall. It is the opposite in Case 3 where the saltwater wedge was completely flushed out much later than in the experiment. The higher the dispersivity ratio, the lower is the transverse dispersivity and the thinner is the mixing zone leading to a longer time of complete removal of the saltwater wedge.

\section{CONCLUSIONS}

In our laboratory-scale study in a saturated porous media made of glass beads with a mean diameter of $0.088 \mathrm{~cm}$, the longitudinal and transverse dispersivities, and their ratio were estimated using a pulse-type fluorescent tracer injection experiment to be $0.07 \mathrm{~cm}$, $0.0025 \mathrm{~cm}$, and $28 \mathrm{~cm}$, respectively. By using these values, the numerical calculation of saltwater intrusion and removal processes after cutoff wall installation, reproduce well the experimental salt concentration changes with time. Our numerical simulation illustrates the fact that accurate estimation of dispersivity is important and the dispersivity ratio $\alpha_{\mathrm{L}} / \alpha_{\mathrm{T}}=10$ usually employed is not always applicable. Although mechanical dispersion in the transverse direction is a much weaker process than in the longitudinal direction, our study demonstrates that the transverse dispersion plays an important role in the reduction of residual saltwater after the cutoff wall installation.

\section{REFERENCES}

C. D. Langevin, D. T Thorne, Jr, A. M. Dausman, M. C. Sukop, and W. Guo 2008. SEAWAT Version 4: A Computer Program for Simulation of Multi-Species Solute and Heat Transport. US Geological Survey Techniques of Water Resources Investigations 6-A22.

Huang, W., C. Smith, D. Lerner, S. Thornton, and A. Oram 2002. Physical modeling of solute transport in porous media: evaluation of an imaging technique using UV excited fluorescent dye. Water Research 36: 1843-1853.

C. Zheng and P. P. Wang 2008. MT3DMS Documentation and User's Guide. U.S. Army Corps of Engineers.

Luyun Jr., R., K. Momii, and K. Nakagawa 2009. Laboratory-scale saltwater behavior due to subsurface cutoff wall. Journal of Hydrology 377: 227-236.

Todd, D.K., and L. W. Mays 2005. Groundwater Hydrology Third Edition. John Wiley \& Sons, Inc.

Contact Information: Roger A. Luyun Jr., Land and Water Resources Division, College of Engineering and Agro-industrial Technology, University of the Philippines Los Baños, Phone/Fax: +6349-536-2387, Email: raluyun1@up.edu.ph 